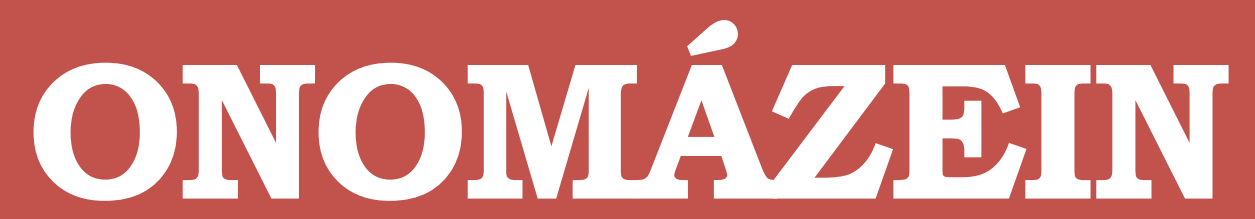

Revista de lingüística, filología y traducción
PONTIFICIA UNIVERSIDAD

\title{
El habla reportada en el discurso de hablantes con afasia sensorial: sus propiedades formales y funcionales
}

Reported Speech in Speakers with Sensorial Aphasia: Its Formal and Functional Properties

\section{Josaphat E. Guillén Escamilla}

Benemérita Universidad Autónoma de Puebla México

\section{(c) $\odot$}

Josaphat E. Guillén Escamilla: Maestría en Diagnóstico y Rehabilitación Neuropsicológica, Facultad de Psicología, Benemérita Universidad Autónoma de Puebla, México | Correo electrónico: jguillen@colmex.mx 


\section{Resumen}

La afasia sensorial es un trastorno del lenguaje provocado por un daño neurológico en el lóbulo temporal del hemisferio izquierdo, donde se ven alteradas todas las formas del lenguaje expresivo. Tradicionalmente, se ha sostenido que estos hablantes retienen la habilidad para comunicarse explotando aquellos recursos que conservan a pesar de la patología. Entre estos recursos se encuentra el habla reportada, que ha sido descrita como una herramienta que les permite a los hablantes afásicos enriquecer sus narraciones e involucrar a sus interlocutores en ellas; sin embargo, aún no contamos con investigaciones centradas en este fenómeno en pacientes hispanohablantes. Por lo anterior, en este trabajo planteamos dos objetivos: (i) analizar si el uso de habla reportada se mantiene en hispanohablantes con afasia sensorial y, de ser el caso, (ii) caracterizar sus propiedades estructurales y funcionales en la producción por parte de estos pacientes. Participaron tres hablantes con afasia sensorial: dos con etiología vascular y uno con etiología tumoral. Todos fueron evaluados empleando el Test de Boston para el diagnóstico de la afasia. Se observó que los pacientes conservan el uso del habla reportada, respetando sus rasgos formales y cumpliendo funciones específicas de compensación e intensificación. Lo anterior sugiere que los pacientes con afasia sensorial son sensibles al déficit que padecen y buscan subsanarlo explotando los recursos que mantienen.

Palabras clave: afasia sensorial; intensificadores; representación; narración; estrategias compensatorias.

\section{Abstract}

Sensory aphasia is a language disorder produced by a neurological damage in left hemisphere's temporal lobe, where it is altered all expressive language form. Frequently, it has been argued that these patients are able to communicate exploiting those resources which they keep even though pathology. These resources include reported speech, which it has been described as a tool that enables it to aphasics enrich his / her narratives to involve his / her interlocutors in it; however, there is still no research focused on this phenomenon in Spanish speakers patients. Thus, in this paper we have two goals: (i) to analyze if our patients keep ability to use reported speech in his / her narrations and, if this is the case, (ii) characterizer 
its formal and functional properties. Our sample is composed of three patients with sensory aphasia, two with vascular etiology and one with tumor etiology. All three were evaluated using Boston Diagnostic Aphasia Examination. It was found that all patients are able to use reported speech, according to their formal features and they use it for accomplish specific functions of compensation and intensification. This suggests that patients with sensory aphasia are aware of their linguistic deficits and thus try to exploit the communicative tools that they keep.

Keywords: sensory aphasia; intensifiers; enactment; narration; compensatory strategies. 


\section{Introducción}

Desde hace unos años a la fecha, la investigación lingüística centrada en los déficits del lenguaje en la afasia ha ido en aumento. Esto se ha visto reflejado, por un lado, en la cantidad de estudios que han aparecido y, por el otro, en la diversificación de los temas y fenómenos que se analizan. Así pues, no es de extrañar que las investigaciones más recientes se alejen de los temas tradicionales — desde los fonéticos hasta los semánticos - y ahora se estén volcando hacia el análisis de fenómenos pragmáticos y discursivos. Ahora bien, más allá de la diversidad, este tipo de estudios ha demostrado que puede ser útil, entre otras cosas, para repensar las clasificaciones tradicionales de las afasias (Pietrosemoli, 1994), para diseñar instrumentos para la evaluación del componente pragmático (Gallardo-Paúls, 2009) y para refinar las características atribuidas al discurso de estos pacientes. A pesar de esto, aún son muchos los fenómenos discursivos que no han sido abordados en el ámbito hispánico y cuyas caracterizaciones provienen solo de la tradición anglosajona. Precisamente, el objeto de estudio que aquí nos interesa es uno de ellos: el habla reportada.

\subsection{El habla reportada}

El recurso del habla reportada (HR) ha sido descrito ampliamente desde una perspectiva formal, es decir, se ha privilegiado la caracterización de sus propiedades estructurales en detrimento de sus propiedades funcionales (cf. Gallucci, 2012). En este sentido, tenemos que puntualizar que, siguiendo a Maldonado (1999: 3554), aquí entenderemos HR como un fenómeno fundamentalmente discursivo y no oracional, o sea, es un acto primordialmente narrativo, ya que en él se habla del "ahí" y el "entonces", en lugar del "aquî" y el "ahora” (Tannen, 1989; Mora y Álvarez, 2003; Hengst y otros, 2005; Mateus, 2005; Shiro, 2007; Galluci, 2012), donde, además, un hablante representa el habla y el pensamiento de otro tiempo, otro lugar y, con frecuencia, otro participante. Así, en el HR se combinan múltiples voces, contextos y metas (Hengst y otros, 2005; Vanderveen, 2014: 15-6).

Regularmente, se distinguen dos tipos generales de HR: (i) estilo directo y (ii) estilo indirecto. En el primer caso, el hablante lleva a cabo la reproducción literal de lo dicho por él o por alguien más, logrando con esto modificar "el sistema de referencias deícticas" (Mal-

1 Entendido grosso modo como: "Las historias sobre los propios hablantes o terceros, presentes o ausentes, las acciones o sucesos ocurridos. Se trata de una sucesión mínima de acontecimientos orientados hacia un final y su complicación; presenta, así pues, carácter temporal y suele tener una evaluación. A la hora de contar se emplea, por lo general, el estilo directo o indirecto libre" (Briz y Albelda, 2013: 310). 
donado, 1999: 3551), que se traslada y se ajusta al momento de la enunciación original. Por otro lado, el estilo indirecto prototípicamente requiere un elemento introductor de cita, la conjunción que, y un verbo dicendi. La diferencia principal es que en estos casos el origo (Bühler, 1967) no se modifica. En una descripción más puntual, Camargo (2004) sostiene que existen diversas formas para representar el habla reportada en la narración oral, de las cuales conviene destacar cuatro: (i) cita directa lingüística (con o sin elemento introductorio), (ii) cita indirecta lingüística (introducida por la conjunción que), (iii) cita no lingüística de paralenguaje (sin elemento introductor y que presenta risas, gritos, interjecciones, etcétera) y (iv) cita no lingüística de gestos (introducida frecuentemente por la locución así $[d e])^{2}$. Aquí nos centraremos en las dos primeras, describiendo sus características formales y funcionales.

\subsubsection{Aspectos formales}

De acuerdo con Gallucci (2012), la estructura prototípica del HR en estilo directo tiende a presentar los siguientes elementos: (i) una expresión introductoria (EI) que contiene (ii) un verbo de reporte, también llamados de comunicación o dicendi; (iii) una cita directa (CD), marcada en la oralidad por medio de recursos prosódicos, y (iv) el contenido citado (CC)3, que reproduce, literalmente o no, uno o varios enunciados.

\section{(1) [El Mi primo me dijo]: [CD [CC "allá nos vemos"]].}

Aunque Maldonado (1999) también señala la existencia de un discurso directo libre que se distingue porque en él se reproducen los enunciados de forma literal, pero sin ningún tipo de verbo u otro elemento gramatical que sirva para introducir la cita y que separe "el texto que corresponde al narrador del que pertenece a los personajes" (Maldonado, 1999: 3275). En estos casos, la única pista con la que contamos son los recursos prosódicos del hablante, ya

2 Las otras son citas: (i) cuasi indirectas, del tipo: según dicen, sí llegan hasta acá, (ii) comentarios metadiscursivos, del tipo: lo que te estaba diciendo hace rato, si ibas a ir o te ibas a quedar, (iii) narración de acción lingüística, del tipo: no dejaba de hablar de él y de su viaje, y (iv) narración de voz, del tipo: y no te dicen nada (Camargo, 2004).

3 Gallucci (2012), en cuanto al contenido de las citas, sostiene que: (i) no necesariamente están constituidas, desde una perspectiva sintáctica, por una oración reportada, sino que pueden estar conformadas por enunciados sin ningún verbo conjugado. (ii) El contenido puede estar formado por fragmentos que no citan palabras reportadas o, más aún, puede estar constituido completamente por estructuras utilizadas para llenar vacíos de información durante el intercambio comunicativo (y me dijo que: bla, bla, bla). (iii) No solo citamos palabras, también reportamos gestos, risas, onomatopeyas e interjecciones. 
que la entonación constituye el parámetro determinante para distinguir entre las dos voces ${ }^{4}$ (Mora, Martínez y Álvarez, 2009).

Por su parte, el HR en estilo indirecto tiende a presentar los siguientes elementos formales: (i) expresión introductoria (EI) que contiene (ii) un verbo dicendi, (iii) una cita indirecta (CI), introducida por la conjunción (iv) que, y (v) el contenido citado (CC) (Gallucci, 2012).

(2) a. [El Juan me dijo] [Cl que [CC quería ir al estadio]].

b. Juan me $1 o_{i}$ dijo [que quería ir al estadioi].

Como vemos, en (2a) la oración encabezada por la conjunción que es una subordinada de objeto directo, ya que puede elidirse anteponiendo el clítico de acusativo al verbo de la oración principal, como en (2b). Así, en estos casos, la cita indirecta está subordinada al verbo de la oración principal.

En resumen, podemos decir que son dos las diferencias fundamentales entre estilo directo y estilo indirecto: (i) el reajuste o cambio del centro deíctico y (ii) el elemento introductor, esto es, siempre que haya un cambio en el origo, aun sin un elemento introductor, estaremos frente a una cita en estilo directo, y cuando exista un elemento introductor, un verbo dicendi y la conjunción que, sin cambio en el origo, estaremos frente a una cita en estilo indirecto 5 (Van der Houwen, 1998; Maldonado, 1999; Gallucci, 2012).

\subsubsection{Aspectos funcionales}

Ya hemos mencionado que la descripción del HR se ha centrado principalmente en la estructura gramatical de las construcciones que la codifican (Besnier, 1992: 163; Gallucci, 2012); no obstante, cada vez son más los estudios interesados en caracterizar sus funciones. Por ejemplo, Van der Houwen (1998) plantea que el estilo directo y el estilo indirecto cumplen con funciones comunicativas distintas; en el primer caso, el hablante trasporta al oyente directamente

4 Para Mora, Martínez y Álvarez (2009: 244) las particularidades prosódicas son una forma en la que los hablantes indican que están citando directamente, esto es, funcionan como claves para contextualizar al HR directa. Para estos autores, el HR directa se caracteriza prosódicamente por: (i) iniciarse con un tono superior al tono previo del discurso, (ii) presentar mayor intensidad que el discurso correspondiente a la voz del emisor y (iii) comenzar con sílabas cortas que gradualmente se alargan hasta la última sílaba (2009: 250).

5 Cabe mencionar que, en el registro coloquial, el HR indirecta también puede aparecer sin ningún tipo de elemento introductor. 
a un contexto comunicativo particular, lo que se puede notar gracias a que el centro deíctico se ha trasladado a ese nuevo escenario. Así, el hablante lleva a otro tiempo al oyente, donde deja de presentar los hechos para cederle la voz a alguno de los participantes de su narración.

En el caso del estilo indirecto, según la propia Van der Houwen (1998), el hablante introduce al oyente en un escenario comunicativo distinto, es decir, el cambio no es abrupto porque el centro deíctico no cambia, aun cuando el tiempo verbal de la expresión introductoria y el de la cita indirecta puedan diferir. En estos casos, el hablante no cede la voz totalmente a ninguno de los participantes de su narración. Así pues, la primera distinción funcional responde al grado de involucramiento del hablante y el oyente con la narración; totalmente involucrados en el primer caso, parcialmente involucrados en el segundo. Finalmente, comparadas con el estilo indirecto, las construcciones en estilo directo pueden identificarse, de manera más sencilla, gracias a que usualmente están enriquecidas con elementos no verbales ostensivos e información paralingüística, como gestos, señas y movimientos corporales (Groenewold, 2015: 136).

Otra función del HR, en general, es que tiende a aparecer cuando el hablante está relatando una anécdota o reportando un pensamiento (Gallucci, 2009; San Martín y Guerrero, 2012); específicamente, se recurre al HR directa en los momentos catárticos de la narración; incluso, en estos casos, se puede prescindir de cualquier elemento introductor (Mateus, 2005). De tal forma, Fernández (2011) explica que la función pragmática que cumple el HR directa e indirecta es ejemplificar; en el primer caso lo hace representando - escenificando-, mientras que en el segundo tiende a utilizarse solo para relatar. En este sentido, de acuerdo con Shiro (2007: 139), "el DD [discurso directo] y las onomatopeyas contribuyen a la dramatización de la producción narrativa, característica que permite mantener la atención del interlocutor durante la ejecución del relato que requiere que el hablante mantenga su turno de habla por un tiempo mayor y contribuyen a la escalada de suspenso que conduce al punto culminante, ayudando así a la organización discursiva del relato". Precisamente, esta parece ser una de las principales funciones del HR en estilo directo en los hablantes con afasia sensorial, como veremos más adelante.

Por último, el HR cumple también con una función modalizadora, esto es, "when a speaker employs convetional brackets to warns us that what he is saying is meant to be taken [...] as mere repeating of words said by someone else, then it is clear that he means to stand in a relation of reduced personal responsability for what he is saying” (Goffman, 1974: 512). Así, la manera en que el hablante elige representar las palabras de otros le permite aliarse con una identidad social preferida o alejarse de la responsabilidad de lo dicho en el enunciado (Irvine, 1996). Así, de acuerdo con Shiro (2012: 122), darles la palabra a algunos de los personajes de la narración nos permite señalar, por un lado, la importancia de los mismos y, por otro, la posición del narrador con respecto al mundo que está construyendo en el relato. 


\subsection{La afasia sensorial}

La afasia sensorial es un trastorno del lenguaje causado por un daño neurológico en el lóbulo temporal del hemisferio izquierdo, específicamente en la circunvolución posterior superior (zona 22 de Brodmann) y otras áreas circundantes (Marquardt y Kiran, 2011). En este tipo de afasia, el factor neuropsicológico afectado es el oído fonemático (Quintanar, Solovieva y León-Carrión, 2011), lo que provoca una alteración en la comprensión del lenguaje y en todas las formas del lenguaje expresivo. El cuadro clínico incluye, entre otras cosas, un déficit léxico profundo (anomia y circunloquios), presencia de parafasias (sustitución de fonemas o palabras) y neologismos (palabras inventadas) que componen al llamado jergón afásico (habla vacía). La repetición y pronunciación de palabras presentan muchas dificultades por la incapacidad de distinguir fonemas (Quintanar, 2002). Tradicionalmente, se ha sostenido que este síndrome viene acompañado de déficits discursivos profundos, en algunos casos tan severos que obstaculizan la comunicación de los hablantes, por lo que han sido considerados incoherentes, tangenciales, no relevantes (Sperber y Wilson, 1986) y no cooperativos (Grice, 1975). No obstante, la investigación en el ámbito hispánico (Pietrosemoli, 1994; Gallardo-Paúls, 2009; Guillén, 2013) ha demostrado que el componente pragmático-discursivo se preserva mejor.

Así pues, en un nivel discursivo, se ha descrito que los hablantes afásicos explotan aquellos recursos que conservan para comunicarse mejor, entre estos elementos se encuentra el HR, ya que le sirve a los pacientes para enriquecer sus narraciones, involucrar a sus interlocutores y como estrategia de simplificación (Goodwin, 2003; Penn, 2007). A pesar de estos resultados, actualmente no contamos con estudios centrados exclusivamente en la afasia sensorial y en pacientes hispanohablantes.

De acuerdo con lo expuesto hasta ahora, creemos que analizar el HR en hispanohablantes con afasia sensorial contribuiría a ampliar la descripción de las habilidades discursivas en esta patología; además, permitiría detallar sus propiedades formales y funcionales y, sobre todo, comprobar su uso como elemento estilístico para enriquecer la narración y como estrategia de simplificación. De este modo, los objetivos que perseguimos en este trabajo son: (i) analizar si el uso de habla reportada se mantiene en hispanohablantes con afasia sensorial y, de ser el caso, (ii) caracterizar sus propiedades estructurales y funcionales.

\section{Antecedentes}

\subsection{Intensificadores y enactment}

Varios autores concuerdan con que el lenguaje afásico se trata de compensación. Goodwin (2003: 10) sostiene que una de las cosas que puede suceder cuando se presenta un trastorno del lenguaje es una reorganización del sistema, donde se explota el uso de aquellos elemen- 
tos que permanecen a pesar de la patología. Por su parte, Penn (2007: 223) destaca que muchas de las conductas que se presentan en la afasia no necesariamente son síntomas afásicos, sino, más bien, son el reflejo de la adaptación del organismo a la nueva condición. Finalmente, Oliveira y Oliveira (2014: 132) apuntan que, si la afasia afecta ciertas estructuras y usos del lenguaje, el hablante que la padece busca otras formas para comunicarse, esto es, crea procesos alternos para poder llevar a cabo la comunicación.

Así pues, en el caso del discurso narrativo, los hablantes con afasia utilizan varios recursos para mejorar la calidad de sus intervenciones, principalmente para dramatizar la narración. A estos recursos se les ha llamado intensificadores, ya que "they convey the narrator's feelings about characters and events and include ritual utterances, phonological intensifiers, lexical items, repetition and gesture, comparatives, syntactic devices such as ellipsis and topicalization, as well direct quotations, asides and sound effects" (Penn, 2007: 232, énfasis agregado). Con estos intensificadores, el hablante es capaz de seleccionar un nivel de detalle para moldear sus ideas, destacar ciertos aspectos para hacer la historia comprensible o emocionante, y alterar secuencias y cambiar perspectivas para representar los sentimientos y pensamientos de otros participantes (Penn, 2007: 232). De tal forma, este tipo de recurso pragmático contribuye a la comunicación del hablante con afasia y muestra que sus intervenciones son paquetes semióticos multimodales (Goodwin, 2003), constituidos por elementos lingüísticos (estructurales), ostensivos (señales deícticas) y paralingüísticos (representaciones gestuales).

Por otra parte, junto con los intensificadores, existen otros recursos compensatorios comunicativos empleados para representar vívidamente los distintos elementos de la narración. Este tipo de recurso ha sido definido como enactment o representación y se refiere al uso de herramientas comunicativas que sirven para mostrar más que describir acciones, participantes y eventos en la interacción comunicativa (Goodwin, 2000, 2003; Wilkinson y otros, 2003; Penn, 2007; Groenewold, 2015). Estas herramientas incluyen diversos elementos comunicativos, como el habla reportada, gestos, movimientos corporales, prosodia, y, en conjunto, pueden representar icónicamente ciertos aspectos de una escena o una acción reportados en la narración (Goodwin, 2003: 7; Penn, 2007: 233; Wilkinson y otros, 2010).

Así pues, el enactment cumple con dos funciones, una pragmática y otra compensatoria. En el primer caso, como estrategia interaccional, ayuda al hablante con afasia a alcanzar sus metas comunicativas, aun cuando se pueda privilegiar el uso de recursos paralingüísticos y no verbales, dados los déficit léxicos y gramaticales que padece (Groenewold, 2015: 8). Al hacer esto, el hablante puede promover la participación activa del interlocutor durante la conversación (Tannen, 1989). En el segundo caso, cuando el hablante con afasia representa lo que los participantes hicieron o dijeron, puede lidiar, de mejor manera, con las demandas contextuales de la conversación. De hecho, esto podría estar reflejando los intentos deliberados del 
hablante para compensar la reducción de sus capacidades lingüísticas en el procesamiento de las tareas narrativas en línea (Penn, 2007: 234).

\subsection{Estudios previos de habla reportada y afasia}

De acuerdo con algunos autores (Hengst y otros, 2005; Ulatowska y otros, 2011), son pocos los estudios centrados en el papel del habla reportada en la afasia; aún más, existen limitaciones metodológicas en este tipo de investigación, entre ellas la más notable es que se ha centrado casi exclusivamente en el inglés (Groenewold, 2015: 11). Sin embargo, en los últimos años, los trabajos sobre este tema han aumentado y se han realizado en otras lenguas como el portugués (Oliveira y Oliveira, 2014) y el holandés (Groenewold y otros, 2014; Groenewold, 2015). En general, se ha aceptado que el HR es un rasgo muy importante de la narración que se preserva en los hablantes con afasia, a pesar del daño profundo en el lenguaje (Ulatowska y Olness, 2003; Hengst y otros, 2005; Penn, 2007; Wilkinson y otros, 2010; Ulatowska y otros, 2011; Oliveira y Oliveira, 2014; Vanderveen, 2014; Groenewold y otros, 2014; Groenewold, 2015). Otro aspecto en el que coinciden estos autores es que los hablantes con afasia utilizan más el HR directa que la indirecta. Por su parte, los estudios contrastivos con hablantes sin daño neurológico no han sido concluyentes; algunos sostienen que no hay una diferencia significativa en el uso de HR (Vanderveen, 2014), otros argumentan que los hablantes con afasia la emplean en mayor medida (Groenewold y otros, 2014; Groenewold, 2015), mientras que otros han encontrado el patrón contrario (Hengst y otros, 2005).

En cuanto a las funciones que desempeña el HR, como ya mencionamos, la mayoría de las investigaciones coinciden en señalar dos: (i) compensar los déficits del lenguaje y (ii) la intensificación de la narración. Así, en palabras de Penn (2007: 232), el HR cumple una función pragmática que sirve como un elemento estilístico que enriquece la narración (intensificador), pero, a su vez, es una estrategia de simplificación que ayuda a evitar cambios (i) de tiempo, (ii) de referencias y (iii) la coordinación o subordinación de enunciados.

En resumen, de acuerdo con los estudios previos, al menos en otras lenguas, el uso de HR se mantiene a pesar de la afasia; sin embargo, no encontramos trabajos centrados en el español que comprueben estas hipótesis. Así pues, nuestra meta es proveer evidencia lingüística que respalde o rechace los resultados antes citados.

\section{Método}

De acuerdo con los objetivos de nuestro trabajo, la metodología que diseñamos tiene como finalidad reflejar, de la forma más natural posible, las características de las producciones 
lingüísticas de los hablantes con afasia sensorial. Así pues, como intentamos describir un fenómeno particular, el del HR, nuestro estudio es puramente descriptivo y, en consecuencia, nuestras hipótesis también lo son.

\subsection{Participantes}

Para nuestro estudio integramos una muestra de tres hablantes mexicanos diagnosticados con afasia sensorial, dos hombres (CL, DM) y una mujer (IE), los tres monolingües del español. Las siglas de identificación de cada paciente fueron elegidas al azar y no corresponden a las iniciales de sus nombres. En cuanto a las condiciones clínicas, los sujetos masculinos adquirieron la afasia debido a un evento vascular cerebral, mientras que la mujer por la extirpación de un tumor en el cerebro; en los tres casos la zona afectada fue la circunvolución posterior superior del lóbulo temporal del hemisferio izquierdo y otras áreas circundantes, lo que provocó un daño en la corteza auditiva primaria y en el oído fonemático. En cuanto al tiempo aproximado de inicio de la enfermedad, al momento de las entrevistas CL tenía 2 meses, DM, 8, e IE, 10. Los tres pacientes no tienen ningún antecedente neurológico o psiquiátrico, tienen lateralidad derecha, el nivel de instrucción promedio es básico y las edades oscilan entre 50 y 65 años. En cuanto a sus ocupaciones, CL era zapatero, DM, ayudante en comercio e IE, ama de casa. Finalmente, el nivel socioeconómico de los tres colaboradores es bajo. Estas características demográficas se concentran en el cuadro 1.

\section{CUADRO 1}

Características demográficas y etiológicas de los participantes

\begin{tabular}{|c|c|c|c|c|c|c|c|}
\hline HABLANTE & SEXO & EDAD & $\begin{array}{l}\text { NIVEL DE } \\
\text { INSTRUCCIÓN }\end{array}$ & ETIOLOGÍA & $\begin{array}{l}\text { TIEMPO } \\
\text { DE } \\
\text { INICIO }\end{array}$ & $\begin{array}{c}\text { NIVEL } \\
\text { SOCIO- } \\
\text { ECONÓMICO }\end{array}$ & $\begin{array}{c}\text { ESCALA DE } \\
\text { SEVERIDAD } \\
\text { (TEST DE } \\
\text { BOSTON) }\end{array}$ \\
\hline $\mathrm{CL}$ & $\mathrm{H}$ & 50 & Básico & Vascular & 2 meses & Bajo & 2 \\
\hline DM & $\mathrm{H}$ & 65 & Básico & Vascular & 8 meses & Bajo & 3 \\
\hline $\mathrm{IE}$ & M & 53 & Básico & Tumoral & 10 meses & Bajo & 4 \\
\hline
\end{tabular}

En principio, no realizamos una estratificación demográfica o etaria específica, pues estas particularidades no desempeñan un papel determinante en la severidad del daño o suponen una variación en las características clínicas de los déficits lingüísticos que se presentan en la afasia (Basso, 2003). La única variable que decidimos considerar es el tiempo de inicio de la enfermedad con la finalidad de determinar si el recurso del HR es algo que se recupera o realmente nunca se pierde. Finalmente, los criterios para la selección de los pacientes fueron los siguientes: 
Criterios de inclusión

- Hablantes adultos monolingües del español.

- Diagnóstico de afasia sensorial o de Wernicke.

- Producción de habla espontánea conectada sin dificultad aparente.

- Puntuación ponderada mayor o igual a 2 en la escala de severidad del Test de Boston.

- Ausencia de daño irradiado a otras zonas que pudiera traer consigo otro tipo de déficit lingüístico, de atención o memoria.

Criterios de exclusión

- Haber sufrido otro ictus después de la aparición del síndrome afásico.

- Padecer tendencias psicóticas o haber presentado episodios psicóticos.

- Falta de interés para la interacción social.

- Tener antecedentes psiquiátricos o neurológicos.

- Haber recibido terapia del lenguaje o neuropsicológica por más de tres meses.

La valoración de los participantes se realizó a partir del Test de Boston para el diagnóstico de las afasias (Goodglass y Kaplan, 1986). En particular, se evaluó el grado de severidad del trastorno afásico y nos concentramos en la aplicación de dos subtests: (i) comprensión auditiva y (ii) expresión oral. El primer subtest nos permitió medir el grado de daño en el oído fonemático y el segundo determinar la facilidad para producir habla espontánea. Como tarea específica, para esta investigación realizamos una entrevista semiestructurada, en la que el médico promovía la producción de habla espontánea por medio de preguntas relativas a la condición del paciente, procurando así la aparición de discurso narrativo.

\subsection{Procedimiento}

Los datos fueron obtenidos en el Hospital General de la Ciudad de México, donde los pacientes asistían a consultas médicas al Servicio de Audiología y Foniatría. Así, después de la aplicación del Test de Boston y las subpruebas, los pacientes regresaban al hospital para iniciar o continuar con su programa de rehabilitación, centrado en las habilidades lingüísticas de los pacientes, principalmente en la comprensión y expresión de lenguaje. El tiempo de terapia es proporcional al tiempo de inicio de enfermedad, esto es, mientras CL iba a comenzar la rehabilitación, DM llevaba al menos 3 sesiones e IE Ilevaba más de seis. Cabe mencionar que las sesiones no son sistemáticas porque los pacientes tienden a dejar de asistir o lo hacen de manera irregular debido a sus condiciones socioeconómicas. 
Así pues, en estas sesiones posteriores se llevaron a cabo las entrevistas semiestructuradas, donde los temas giraban en torno a la condición de los pacientes, su trabajo, su familia y, en general, a las actividades que realizan durante el día; todo esto sirvió para promover la aparición de muestras de discurso narrativo. Por su parte, los pacientes y sus familiares fueron informados sobre la naturaleza de nuestra investigación, así como del manejo ético que se le daría a sus datos, por lo que decidieron consentir, de manera expresa, la videograbación de las entrevistas y el uso de ellas con fines meramente de investigación lingüística. Las grabaciones se hicieron de manera individual dentro del consultorio, con la presencia del médico, el paciente, otro investigadory, ocasionalmente, una enfermera.

En general, la duración de las entrevistas no estaba predeterminada, pues dependía de la disposición que tuviera el paciente para conversar. Cuando mostraba desinterés, el médico redirigía la conversación ya fuera cambiando el tema o regresando a alguno donde hubiera tenido mayor participación. La longitud oscila entre los quince y treinta minutos, aproximadamente. Después de realizar las entrevistas, dimos paso a su tratamiento informático. Para la transcripción de los datos utilizamos el programa informático ELAN (Sloetjes y Wittenburg, 2008) y el sistema de etiquetado del Grupo Val.Es.Co. (Briz y Grupo Val.Es.Co., 2002a, 2002b, 2003). Una vez transcrito y etiquetado, nos dimos a la tarea de rastrear los usos de HR atendiendo a las características formales que se describieron líneas arriba. En casos dudosos, regresamos a la grabación para determinar si se trataba de HR o no. Finalmente, dividimos el HR en directa e indirecta en cada paciente y para su inclusión ejemplar dentro del texto decidimos conservar las etiquetas del Grupo Val.Es.Co.

\section{Resultados}

\subsection{Habla reportada directa}

De acuerdo con el análisis, Ios hablantes recurren más al HR directa que a la indirecta. En el caso del hablante CL, tuvo 17 usos de HR, 13 de ellos en estilo directo. De estos 13 usos, 10 fueron presentados sin ningún tipo de verbo o partícula introductoria. Por su parte, el hablante DM empleó 33 veces el HR, 27 de ellas en estilo directo. De estas 27 ocasiones, solo 3 fueron presentadas sin partícula introductoria. Finalmente, IE empleó 25 veces el HR, 24 de ellas en estilo directo y solo en una ocasión no presentó partícula introductoria. Por otra parte, cuando sí se utilizó una partícula introductoria, en todos los casos la única que apareció fue el verbo decir. Finalmente, en el uso de autocitas hubo diferencias entre los hablantes; CL solo se autocitó tres veces, mientras que DM lo hizo en 21 ocasiones e IE en 14 oportunidades. Los resultados se encuentran concentrados en el cuadro 2.

Como podemos ver en los siguientes ejemplos, las muestras de HR directa son introducidas por el verbo decir y los centros deícticos se modifican; además, cuentan con otro tipo de 


\section{CUADRO 2}

Uso de HR directa

\begin{tabular}{ccc|c|c|c} 
HABLANTE & TOTAL HR & HR DIRECTA & AUTOCITAS & $\begin{array}{c}\text { SIN PARTICULA } \\
\text { INTRODUCTORIA }\end{array}$ & $\begin{array}{c}\text { TIEMPO DE } \\
\text { EVOLUCIÓN }\end{array}$ \\
\hline $\mathrm{CL}$ & 17 & 13 & 3 & 10 & 2 meses \\
\hline $\mathrm{DM}$ & 33 & 27 & 21 & 3 & 8 meses \\
\hline IE & 25 & 24 & 14 & 1 & 10 meses \\
\hline
\end{tabular}

intensificadores, como los adverbios exclamativos cómo y qué, en (3) y (4), y la interjección jay! en (5). De tal forma, nuestros hablantes respetan la estructura formal del HR directa, según lo visto anteriormente:

(3) CL: pss / es que luego digo ya agarraron allí y digo / ¡CÓMO! / noo / está mal / síi / no

(4) DM: me dice [de cosas] a mí y le digo / noo / yo soy hombre iQUÉ!

(5) IE: le digo / iAY! este te dije se me olvidó decirlo comple- poquín ${ }^{6}$

Ahora bien, los casos en los que el HR directa se presenta sin elemento introductor tienen características muy particulares; en ellos, los hablantes tienden principalmente a representar las acciones que están narrando, esto es, le muestran al interlocutor la escena tal y como sucedió y lo hacen partícipe, lo envuelven en ese nuevo contexto:

(6) CL: y la otra puss / váyase na por favor señora / mejor nos vamos aquí con la señora / por eso siempre iba yo

CL: pues estoy bien ¿no? / nomás que luego voy demulato pérese tantito / ya va

(7) (a) DM: y los amigos / mire / siempre jora / vente a comer!

(b) DM: una señora me da / órale pa’ que se ponga [ropa]

(8) IE: yo aprendí a hablar / dime ¿cómo dice aquí?

Así, el contenido de las citas directas presenta las acciones tal y como acontecieron ajustándose al tiempo, lugar y participantes. Los ejemplos más claros son (7) y (8); en (7a) el hablante

6 Brevemente, en la convención de etiquetado, las cursivas señalan el contenido citado, las MAYúsCULAS corresponden a pronunciación pesada o enfática y las diagonales indican pausas cortas, inferiores a medio segundo. 
representa literalmente la forma en que los amigos lo invitan a comer, y en (7b) la manera en que una señora le ofrece ropa. En (8) sucede lo mismo, IE representa la forma en que le enseñaron a hablar de nuevo. Un aspecto digno de destacar es que, en estos casos, el contenido de la cita directa cumple con una función conativa (Jakobson, 1981), esto es, el hablante citado está interpelando al oyente para que actúe o reaccione según lo solicitado, por eso lo que está codificado en la cita son actos exhortativos: preguntas, ruegos, mandatos o interjecciones que cumplen con esta función. En nuestros datos, la gran mayoría del contenido citado corresponde a estos ejemplos, lo que podría estar indicando que una de las funciones del HR directa es precisamente representar este tipo de actos.

Por otra parte, otro fenómeno que debemos destacar, y que se presentó exclusivamente en el estilo directo, es que los hablantes no solo representan textualmente la intervención de un interlocutor en particular, sino que, incluso, presentan diálogos enteros entre dos o más participantes, como en los siguientes ejemplos:

(9) CL: la mejor de queda en trurrarra / téngame los cien / sí señora / y ahí la dejo

(10) DM: hasta decía / por FAVOR / déjenle lo que tiene / está [usted] bien pero para qué les dice / digales que está bien pos más [le roban]

(11) IE: yo sí me daba cuenta lo que estaba diciendo pero yo le decía / ¡AY ya nada! / ¿CÓMO nada?!

Los casos de (9) a (11) también son ejemplos de representación, pues los hablantes están presentando, más que eventos, escenas pasadas completas como si estuvieran ocurriendo justo en el momento de la enunciación, y lo hacen ya no solo a partir de intensificadores, sino primordialmente a partir de la escenificación de diálogos entre distintos hablantes y distintos destinatarios. El caso más representativo es (10), reproducido abajo como (12):

(12) [A dirigiéndose a los familiares del paciente]

A: por favor, déjenle lo que tiene

[A dirigiéndose al paciente DM]

A: está [usted] bien, pero para qué les dice, dígales que está bien pos más le roban

Justo en este tipo de casos resultan más útiles los recursos prosódicos, pues le permiten a los hablantes segmentar la cita, señalando hasta dónde corresponde la intervención de cada participante y, así, proveer la estructura de diálogo.

Así pues, los ejemplos presentados de (3) a (11) muestran que los hablantes respetan la estructura del HR directa, esto es, no es indispensable que aparezca una partícula introductoria, sin embargo, lo que sí es condición sine qua non es el cambio en el sistema deíctico de referencias del hablante. Aún más, las citas directas tienen una configuración prosódica par- 
ticular, lo que permite distinguirlas incluso si no están acompañadas por una partícula introductoria. En consecuencia, desde una perspectiva formal, nuestros hablantes son capaces de utilizar convencionalmente el HR directa, ciñéndose a rasgos sintácticos y suprasegmentales.

Ahora bien, en cuanto a las funciones que desempeña el HR directa, estas coinciden con las expuestas en el apartado anterior, esto es, sirve para (i) compensar los déficits lingüísticos e (ii) involucrar al interlocutor en la narración. En el primer caso, podemos retomar los ejemplos de (9) a (11), donde los hablantes presentan un evento discursivo, cediéndole la voz a otros participantes y, al hacerlo, simplifican las dificultades sintácticas que supone la alternancia de referentes y de tiempos, principalmente. Más aún, al reproducir diálogos completos entre más de dos hablantes, los pacientes prescinden de cualquier complejidad estructural para especificar los cambios de emisor, destinatario y tiempo, y solo deben recurrir a rasgos prosódicos particulares para hacerlo.

En el segundo caso, cuando los hablantes recurren al HR directa y a otros intensificadores (interjecciones, gestos, señas y recursos prosódicos), como en los casos de (3) a (8), lo que hacen es dramatizar el episodio narrativo buscando involucrar a sus interlocutores en él. En otras palabras, nuestros hablantes representan eventos pasados y los muestran como si estuvieran ocurriendo en el "aquí" y en el "ahora” de la enunciación, frente a los ojos de su audiencia, envolviéndola y haciéndola partícipe de la narración, como testigos de los hechos más que como simples oyentes. En breve, podemos plantear que todos estos recursos son empleados por nuestros pacientes porque les permiten llamar y retener la atención del interlocutor, ya que hacen ostensiva la importancia del episodio discursivo que están presentando en forma de cita directa.

Finalmente, otra función del HR directa es que tiende a presentarse en los puntos álgidos de la narración y cuando los acontecimientos le atañen directamente al hablante, como en el caso de (7), (8), (10) y (11), principalmente. Aunque debemos mencionar que esto último puede deberse a la naturaleza de la entrevista, ya que toda la interacción gira en torno a la perspectiva del paciente.

\subsection{Habla reportada indirecta}

El HR indirecta tuvo un uso periférico en nuestros hablantes, pues solo apareció en 11 ocasiones distribuida de la siguiente forma: CL la utilizó 4 veces, 3 de ellas con el verbo decir y una con opinar, y del total se autocitó en dos ocasiones. En el caso de DM, utilizó en 6 ocasiones el estilo indirecto, todas ellas con el verbo decir; en ningún caso se autocitó y, del total, una vez omitió la conjunción que pospuesta al verbo. Finalmente, IE utilizó el estilo indirecto en una ocasión y fue para autocitarse. En el cuadro 3 presentamos estos resultados. 


\section{CUADRO 3}

Uso de HR indirecta

\begin{tabular}{c|c|c|c|c|c} 
HABLANTE & TOTAL HR & $\begin{array}{c}\text { HR } \\
\text { INDIRECTA }\end{array}$ & AUTOCITAS & $\begin{array}{c}\text { SIN CONJUNCIÓN } \\
\text { QUE }\end{array}$ & $\begin{array}{c}\text { TIEMPO DE } \\
\text { EVOLUCIÓN }\end{array}$ \\
\hline $\mathrm{CL}$ & 17 & 4 & 2 & 0 & 2 meses \\
\hline $\mathrm{DM}$ & 33 & 6 & 0 & 1 & 8 meses \\
\hline IE & 25 & 1 & 1 & 0 & 10 meses \\
\hline
\end{tabular}

(13) CL: sí / ella opina que me- que es usted / puss por favor no / nomás paquito

(14) DM: me ha dicho que ya soy muerto / ya nomás me espero

(15) IE: yo dije que ustedes son los que me han dicho

Como se muestra en los ejemplos de (13) a (15), los hablantes tienden a respetar la estructura del HR indirecta, según lo que presentamos en la introducción, esto es, está compuesta por una Expresión Introductoria, un verbo dicendi acompañado de la conjunción que, una Cita Indirecta y el Contenido Citado:

(16) [El sí, ella opina] [Cl que [CC me- que es usted]].

(17) [El me ha dicho] [Cl que [CC ya soy muerto]].

(18) [El yo dije] [Cl que [CC ustedes son los que me han dicho]].

En cuanto a las funciones que desempeña el estilo indirecto, podemos señalar una: la modalización, sea para alejarse o para responsabilizarse de lo dicho. En el primer caso, el hablante puede introducir "otra voz", distinta a la suya, para deslindarse de lo que se dice, como en (19); en el segundo caso, el hablante se autocita, con lo que se hace totalmente responsable de lo dicho, y no solo eso, además se alía con el interlocutor como un recurso de atenuación (Briz y Albelda, 2013). Generalmente, cuando tiene este matiz atenuante, lo que se cita es un cumplido o un halago buscando un beneficio, como en (20):

(19) DM: el hermano este que es escuincle porque es escuincle / (es)tá viejo también / ese dice que estoy reloco

(20) CL: yo le digo [a ella] que usted me puede ayudar usted / si usted me puede aler las mecuás luego paremuá / tandi fen de usted da

Así, en (19) el hablante se aleja de lo afirmado utilizando (i) el HR indirecta, (ii) restando credibilidad al autor de lo dicho porque es escuincle y (iii) con el uso de pronombre ese, en lugar de él. 
En el caso de (20), el hablante se hace totalmente responsable de lo dicho, dado que (i) se autocita y, a la vez, (ii) crea empatía con el interlocutor, ya que le deja claro que está convencido de que lo puede ayudar. En conclusión, siguiendo a Besnier (1992: 162), podemos sostener que estas citas indirectas se fusionan con el resto del discurso, permitiendo una mayor manipulación del significado y la interpretación de la cita, y esta responsabilidad queda en boca del paciente.

\section{Discusión}

Antes que nada, debemos advertir que los resultados de nuestra investigación no pueden considerarse concluyentes 7 per se, principalmente porque han sido obtenidos a partir de la muestra de tres hablantes, sin embargo, si los consideramos en conjunto con los de los estudios previos, podríamos comenzar a proponer ciertas hipótesis acerca del rol que desempeña el HR en la producción discursiva de hispanohablantes con afasia sensorial.

Como punto de partida, estábamos interesados en investigar si el HR se mantenía en hispanohablantes con afasia sensorial, tal y como ha sido descrito en otras lenguas, y, como segundo objetivo, buscamos caracterizar sus propiedades formales y funcionales, en específico comprobar su papel como elemento intensificador de la narración y como estrategia de simplificación. Así pues, de acuerdo con los resultados de nuestro análisis, en principio podemos postular dos cosas: (i) los pacientes mantienen el recurso del HR respetando sus propiedades formales, tanto estructurales como suprasegmentales, y (ii) las funciones que desempeña en el discurso de nuestros hablantes coinciden con las descritas en los estudios previos. En este punto, nos interesa hacer algunas precisiones sobre estos dos aspectos.

En primer lugar, hemos señalado antes que los pacientes indican el paso al estilo directo, al menos, por medio de dos elementos formales: (i) la modificación en el sistema deíctico de referencias y (ii) el contorno melódico particular que configura a la cita directa. En este sentido, el hecho de que estos dos rasgos se mantengan nos permite reiterar lo que ya hemos dicho en otros lados acerca de que los hablantes con afasia sensorial tienden a conservar, por un lado, la sensibilidad pragmática para manejar, de forma convencional, la deixis temporal, referencial y espacial (Guillén y Morales, 2016). Y, por otro, que la entonación y prosodia son de los aspectos funcionales que persisten consistentemente en esta patología, aun cuando los demás mecanismos lingüísticos puedan estar profundamente dañados (Guillén, 2013; Mendo-

7 Sin embargo, desde una perspectiva neuropsicológica, existen algunas posturas radicales, como la ultracognitiva (Caramazza, 1986; McCloskey, 1993), que sostienen que los estudios basados en un solo paciente proveen la única fuente fiable para inferir, a partir de la patología, el funcionamiento natural de los mecanismos cognitivos, entre ellos, los lingüísticos. 
za y Guillén, 2016). Así pues, lo que queremos destacar es que estos dos rasgos parecen resistir el proceso lingüístico de desintegración que se presenta en la afasia.

En segundo lugar, nuestros pacientes emplean el HR para "decir" lo que ellos mismos no pueden, esto es, cuando recurren al estilo directo recuperan directamente de la memoria episódica ${ }^{8}$ la cita completa, como un todo, más que construirla en línea (Martin y Slevc, 2012). Al hacerlo de esta manera, se ahorran los procedimientos gramaticales y cognoscitivos que ello supone. La evidencia para conjeturar esto proviene de dos fuentes. Por un lado, ya se ha dicho que la memoria declarativa ${ }^{9}$ se mantiene relativamente intacta en este tipo de afasia, principalmente cuando se accede a ella de manera inconsciente, cuando el hablante no está realizando una tarea lingüística muy específica y no se está monitoreando (Guillén, 2016). Por otro lado, la segunda fuente es una perceptual y hasta cierto punto impresionista, pero no por ello menos válida: cuando los hablantes emplean el estilo directo desaparecen, casi por completo, las parafasias y los neologismos, esto es, cesan los problemas léxicos y parece que estamos frente a otro hablante. En resumen, estos dos elementos en conjunto podrían respaldar la hipótesis de que algunos aspectos discursivos son paquetes de información completa, conformados por parcelas discursivas más que por estructuras sintácticas oracionales individuales (Hopper, 1998; Townsend y Bever, 2001). Además, también podrían estar reflejando los intentos deliberados del hablante para compensar la reducción de sus capacidades lingüísticas en el procesamiento de las tareas narrativas en línea (Penn, 2007: 234).

Otro aspecto derivado de nuestro análisis es que los pacientes no solo conservan el HR como el único recurso de intensificación, sino que, también, emplean otros elementos como: interjecciones, gestos, pronunciación enfática y la representación de diálogos completos. En conjunto, todos estos recursos le permiten al hablante hacer más dramática su narración, seleccionar un nivel de detalle, enfatizar ciertos rasgos para hacer la historia más emocionante y, con todo esto, mantener la atención de su(s) interlocutor(es). En estos casos, los pacientes muestran una preferencia para emplear el discurso directo libre, pues les permite trasladar a sus interlocutores directamente a un contexto comunicativo particular.

Por otra parte, pudimos comprobar que el estilo directo es preferido sobre el indirecto, lo que abona a la tesis de que este último es la opción marcada (Bevavent Payá, 2003; Gallucci, 2009; Fernández, 2011; San Martín y Guerrero, 2012). Ahora bien, en el caso específico de nuestros pacientes, esta preferencia puede estar justificada porque el estilo indirecto exige la presencia de más recursos formales y supone una mayor complejidad sintáctica, complejidad con la que no

8 La memoria episódica se define, a grandes rasgos, como el sistema que nos permite recordar experiencias pasadas (Tulving, 1972, 2002). Por tal motivo, también se le conoce como memoria autobiográfica.

9 Cabe recordar que la memoria episódica forma parte del sistema de memoria declarativa. 
pueden lidiar nuestros pacientes. Con todo, el estilo indirecto cumple con la función de modalizar la intervención del paciente, sea para alejarse o para responsabilizarse de lo dicho, en cuyo caso comúnmente viene acompañado de un matiz atenuante. Este resultado también es consistente con los estudios previos en población sin daño neurológico (Irvine, 1996; Shiro, 2012).

Finalmente, en cuanto a las diferencias entre nuestros participantes, podríamos decir que el tiempo de inicio de enfermedad es un factor a considerar, pues el hablante que tenía menos tiempo con su nueva condición presenta características particulares: (i) utiliza menos el HR y (ii) prescinde consistentemente de la partícula introductoria en el caso de estilo directo. Así, podemos suponer que con el paso del tiempo, y con ayuda de la rehabilitación, el recurso del HR se utiliza en mayor medida. No obstante, el hecho de que se emplee en las etapas más tempranas de la enfermedad podría estar indicando que, en realidad, no se pierde, más bien solo se ve minada en algunos aspectos.

\section{Conclusiones}

De acuerdo con lo expuesto en el apartado anterior, podemos adelantar las siguientes conclusiones. En primer lugar, tenemos que destacar que los pacientes con afasia sensorial tienden a conservar, incluso en las etapas más tempranas de la enfermedad, el recurso del HR. Al hacerlo, respetan sus rasgos formales y lo utilizan para cumplir funciones particulares, por lo que su uso comparte varios de los rasgos que han sido descritos en hablantes sin patología, entre ellos que el estilo directo es empleado más comúnmente y que el verbo dicendi más frecuente para introducir el HR es decir.

En cuanto a las funciones particulares que desempeña el HR directa, son dos: (i) como intensificador y (ii) como estrategia de simplificación. En este sentido, el hablante emplea las autocitas para cumplir principalmente con una función de intensificador, pues le permite representar vívidamente la escena que está describiendo y de la que es protagonista.

Por su parte, la función del estilo indirecto es actuar como una marca modal que representa la actitud del hablante con respecto a lo que dice, sea para deslindarse o comprometerse con lo que dice y le permite aliarse con una identidad social preferida. En cuanto a los rasgos prosódicos, estos se mantienen relativamente intactos, ya que en muchas ocasiones son el único indicio para señalar el paso al estilo directo. Esto puede respaldar la hipótesis de que la prosodia en la afasia sensorial se mantiene casi sin daño debido a su carácter altamente funcional. En tanto que el contenido de la cita, al menos en nuestros pacientes, además de incluir gestos, risas, onomatopeyas e interjecciones (Gallucci, 2012), codifica actos exhortativos, fundamentalmente mandatos en modo imperativo.

En breve, consideramos que nuestros pacientes echan mano de aquellos elementos que conservan y explotan su uso porque les ayudan a subsanar los déficits lingüísticos a los que 
se enfrentan, todo esto con la intención de comunicarse de la mejor manera posible para ellos. No obstante, hay que considerar que este es un primer acercamiento al uso y funciones del HR en la afasia sensorial en hispanohablantes, por lo que estas conclusiones son presentadas como hipótesis interpretativas, a la espera de ser corroboradas con más investigaciones con muestras de hablantes más amplias. También son necesarios más estudios interesados en caracterizar, de forma exhaustiva, los recursos prosódicos empleados en el HR, así como trabajos contrastivos con hablantes sin patología.

Finalmente, coincidimos con Holland (1977: 173) cuando afirma que los hablantes con afasia se comunican mejor de lo que hablan.

\section{Bibliografía citada}

Basso, A., 2003: Aphasia and Its Therapy, Oxford: Oxford University Press.

Benavent PayA, E., 2003: “¿Por qué contamos nuestras historias cotidianas en estilo directo?” en N. Delbeque (ed.): Aproximaciones cognoscitivo-funcionales al español Foro Hispánico 23, Ámsterdam: Rodopi, 11-20.

Besnier, N., 1992: "Reported speech and Affect on Nukulaelae Atoll” en J. H. Hilly J. T. IRvine (eds.): Responsability and Evidence in Oral Discourse, Cambridge: Cambridge University Press, 161-181.

Briz, A., y Grupo Val.Es.Co, 2002a: Corpus de conversaciones coloquiales, Madrid: Arco-libros.

Briz, A., y Grupo Val.Es.Co, 2002b: "La transcripción de la lengua hablada: el sistema del Grupo Val.Es.Co”, Español Actual 77-78, 57-86.

Briz, A., y Grupo Val.Es.Co, 2003. “Un sistema de unidades para el estudio del lenguaje coloquial”, Oralia 6, 7-61.

BRIz, A., y M. AlbeldA, 2013: "Una propuesta teórica y metodológica para el análisis de la atenuación lingüística en español y portugués. La base de un proyecto en común (ES.POR.ATENUACIÓN)", Onomázein 28, 288-319.

BüHLER, K., 1967: Teoría del lenguaje, Madrid: Selecta.

CAmARgo, L., 2004: "El estilo directo y el estilo indirecto. De las gramáticas del español al análisis del discurso y la pragmática” en Actas del V Congreso de Lingüística General, vol. I, Madrid: Arco/Libros, 479-489.

Caramazza, A., 1986: "On drawing inferences about the structure of normal cognitive systems from the analysis of patterns of impaired performance: The case for single-patient studies", Brain \& Cognition 5, 41-66. 
Fernández, M. F., 2011: Uso del discurso directo e indirecto en el habla de Mérida. Tesis de Licenciatura, Universidad de Los Andes en Mérida.

Gallardo-Paúls, B., 2009: "Valoración del componente pragmático a partir de datos orales", Revista de Neurología 48 (2), 57-61.

GallucCl, M. J., 2009: “Nos fuimos a la quebrada y mi mamá: ‘Estaban lanzándose por la quebrada?’, ‘¡No, mamá!’, ‘iClaro que sí!’”, Núcleo 26, 75-98.

Galluccl, M. J., 2012: "Estilo directo e indirecto en interacciones orales: Estado de la cuestión en el ámbito hispánico”, Boletín de Filología XLVII (2), 205-233.

Goffman, E., 1974: Frame Analysis: An Essay on the Organization of Experience, New York: Harper and Row.

Goodglass, H., y E. Kaplan, 1986: La evaluación de la afasia y trastornos relacionados, 2. ${ }^{\text {e ed., Ma- }}$ drid: Panamericana.

Goodwin, C., 2000: “Gesture, aphasia, and interaction” en D. McNeILl (ed.): Language and Gesture, Cambridge: Cambridge University Press, 84-98.

Goodwin, C. (ed.), 2003: Conversation and Brain Damage, Oxford: Oxford University Press.

Grice, H. P., 1975: "Logic and conversation" en P. Cole y J. L. Morgan (eds.): Syntax and Semantics 3: Speech Acts, NY: Academic Press, 41-58.

Groenewold, R., 2015: Direct and indirect speech in aphasia: studies of spoken discourse production and comprehension. Tesis doctoral, Universidad de Groningen.

Groenewold, R., R. Bastiaanse, L. Nickels, M. Wieling y M. Huiskes, 2014: "The effects of direct and indirect speech on discourse comprehension in Dutch listeners with and without aphasia", Aphasiology 28 (7), 862-884.

Guillén, J. E., 2013: El uso de marcadores discursivos y las funciones que desempeñan dentro de las producciones lingüísticas de pacientes con afasia de Wernicke. Un estudio contrastivo. Tesis doctoral, El Colegio de México.

Guillén, J. E., y J. Morales, 2016: "La deixis en la competencia comunicativa de pacientes con afasia de Wernicke”, Graffylia 22, 103-112.

Hengst, J. A., S. R. Frame, T. Neuman-Stritzel y R. Gannaway, 2005: “Using Others' Words: Conversational Use of Reported Speech by Individuals with Aphasia and Their Communication Partners", Journal of Speech, Language \& Hearing Research 48, 137-156. 
Holland, A., 1977: "Some practical considerations in the treatment of aphasic patients" en M. Suluivan y M. Kommers (eds.): Rationale for adult aphasia therapy, Omaha, NE: University of Nebraska Press, 167-180.

Hopper, P. J., 1998: "Emmergent Grammar" en M. Tomasello (ed.): The New Psychology of Language, Mahwah, NJ: Erlbaum Associates, 155-175.

IRVINE, J. T., 1996: "Shadow conversations: The indeterminacy of participant roles" en Michael SilversteIn y Greg URban (eds.): Natural histories of discourse, Chicago: The University of Chicago Press, 131-159.

JAKobson, R., 1981: Ensayos de Lingüística General, Barcelona: Seix Barral.

Maldonado, C., 1999: "Discurso directo y discurso indirecto" en Ignacio Bosque y Violeta Demonte (eds.): Gramática descriptiva de la lengua española, vol. 3, Madrid: Espasa Calpe, 3551-3595.

Marquardt, T., y S. KiRAN, 2011: "Acquired neurogenic language disorders" en R. Gilliam, T. Marquardt y F. MARTIn (eds.): Communication sciences and disorders, Boston: Jones and Bartlett, 271-292.

Martin, R., y R. L. SLEVC, 2012: “Memory Disorders and Impaired Language and Communication” en R. PeAch y L. SAPhiro (eds.): Cognition and Acquired Language Disorders, EE. UU.: Mosby, 183-210.

Mateus, L., 2005: El estilo directo e indirecto como estrategias narrativas en el habla de Caracas. Tesis de Licenciatura, Universidad Central de Venezuela, Caracas.

McCloskey, M., 1993: "Theory and evidence in cognitive neuropsychology: A 'radical' response to Robertson, Knight, Rafal, and Shimamura (1993)", Journal of Experimental Psychology: Learning, Memory, and Cognition 19, 718-734.

MendozA, É., y J. E. Guillén, 2016: "Sobre la imbricación de las funciones de la entonación y marcadores discursivos en la afasia de Wernicke”, Estudios de Lingüística Aplicada 63, 133-152.

Mora, E., y A. Álvarez, 2003: "Indirect discourse and its acoustic/prosodic characteristics in Venezuelan Spanish" en M. J. Solé, D. Recasens y J. Romero (eds.): Proceedings of the 15th International Congress of Phonetic Sciences, Barcelona: Causal Productions, 571-574.

Mora, E., H. Martínez y A. Álvarez, 2009: “Características acústico-prosódicas del discurso reportado en el español de Venezuela”, Estudios de Fonética Experimental 18, 237-252.

OliveIRA, L. M., y M. M. OliveIRA, 2014: "The use of direct reported speech by a person with aphasia to construct a narrative", Revista CEFAC 16 (1), 131-146.

Penn, C., 2007: "Cultural dimensions of aphasia: Adding diversity and flexibility to the equation" en M. Ball y J. Damico (eds.): Clinical Aphasiology: Future Directions, Hove, UK: Psychology Press, 221-244. 
Pietrosemoli, L., 1994: “Coherencia y cohesión en el discurso afásico”, Lengua y Habla 1, 78-88.

Quintanar, L., 2002: "La escuela neuropsicológica soviética”, Revista Española de Neuropsicología 4 (1), 15-41.

Quintanar, L., Y. Solovieva y J. León-Carrión, 2011: Evaluación neuropsicológica clínica de las afasias Puebla-Sevilla, México: Benemérita Universidad Autónoma de Puebla, BUAP.

San Martín, A., y S. Guerrero, 2012: "Discurso referido y oralidad en el corpus PRESEEA de Santiago de Chile", comunicación presentada en el Coloquio Interacción y manifestaciones discursivas en el diálogo oral, Santiago de Chile.

SHIRO, M., 2007: La construcción del punto de vista en los relatos orales de niños en edad escolar. Un análisis discursivo de la modalidad, Caracas: Universidad Central de Venezuela.

SHIRO, M., 2012: "Y entonces le dijo... la representación del habla en las narraciones de niños venezolanos”, Boletín de Lingüística XXIV, 119-143.

Sloetjes, H., y P. Wittenburg, 2008: "Annotation by category - ELAN and ISO DCR" en Proceedings of the 6th International Conference on Language Resources and Evaluation.

Sperber, D., y D. Wilson, 1986: Relevance: Communication and Cognition, Oxford: Basil Blackwell.

TAnnen, D., 1989: Talking Voices: Repetition, Dialogue, and Imagery in Conversational Discourse, Cambridge: Cambridge University Press.

Townsend, D., y T. Bever, 2001: Sentence Comprehension. The Integration of Habits and Rules, Massachusetts: MIT Press.

Tulving, E., 1972: "Episodic and semantic memory" en E. Tulving y W. Donaldson (eds.): Organization and memory, New York: Academic Press, 381-403.

TUlving, E., 2002: “Episodic memory: From mind to brain”, Annual Review of Psychology 53 (1), 1-25.

Ulatowska, H. K., y G. S. Olness, 2003: "On the nature of direct speech in narratives of African Americans with aphasia”, Brain and Language 87, 69-70.

Ulatowska, H. K., B. Reyes, T. Olea Santos y C. Worle, 2011: "Stroke narratives in aphasia: The role of reported speech", Aphasiology 25 (1), 93-105.

Van der Houwen, F., 1998: "Organizing discourse. Direct and Indirect Speech in Mexican Spanish", Linguistics in the Netherlands 15, 123-134.

VAnderveen, N. E., 2014: "The use of reported speech in the interactions of individuals with traumatic brain injury". Tesis de maestría, Universidad de Iowa. 
Wilkinson, R., S. BeEke y J. Maxim, 2003: "Adapting to Conversation: on the Use of Pro-Forms by Aphasic Speakers in the Construction of Turns at Talk" en C. Goodwin (ed.): Conversation and Brain Damage, Nueva York: Oxford University Press, 59-89.

Wilkinson, R., S. Beeke y J. Maxim, 2010: "Formulating actions and events with limited linguistic resources: enactment and iconicity in agrammatic aphasic talk", Research on Language \& Social Interaction 43 (1), 57-84. 